保健物理, 28, 31 40（1993）

\title{
ORIGINAL
}

\section{Organ Doses and Effective Dose as a Function of Organ Depths under Body Surface for External Photon Exposure}

\author{
Sangduk SA ${ }^{*, * 1}$ Michio YoshizaWA ${ }^{* *, * 2}$ and Masakuni NarITA ${ }^{* * * * 1}$
}

(Received July 1, 1992)

(Accepted September 28, 1992)

\begin{abstract}
A simplified method in which the equivalent doses in tissue or organs and effective dose for external photon exposure according to the ICRP 1990 Recommendations could be approximated by a single empirical expression is proposed. In this method, the position of an organ is assumed to be the main factor that determine its dose for external photon irradiation. Many organ depths with different body size for ages 1, 5, 10 years and adults were determined using the cross-sections of mathematical organ descriptions, and effective depth for approximating effective dose was obtained with the organ depths assigned to tissue weighting factors.

Compared with the precised data for adults by the Monte Carlo method, the simple expression gives good approximates for most of deep organs and effective dose within about $20 \%$ except some organs, especially for lateral geometry. It was found that irradiation geometry affects the variations in age-dependent effective dose, and that the effective depths for ages $1,5,10$ years, normalized to the adults depth, are directly proportional to the cubic root of the body weight. The usefulness of the concept of effective depth was also discussed with regard to the specified point of effective does in the body, obtained from the combination of the depths for the three typical geometries.
\end{abstract}

KEY WORDS: organ dose, effective dose, tissue weighting factors, organ depth, effective depth, specified point, irradiation geometry

\section{INTRODUCTION}

The International Commission on Radiological Protection (ICRP) has introduced a single equivalent dose quantity-effective dose equivalent, now renamed as effective dose-for the purpose of quantitative estimate of the radiation exposure risk. The new recommendations published as ICRP Publ. $60^{1)}$ has made significant changes in

* 史 相徳, ** 吉沢道夫, $* * *$ 成田正邦

*1 Faculty of Engineering, Hokkaido University; Kita 13 Nishi 8, Kita-ku, Sapporo 060. 北海道大学工学部原子工学科; 札幌市北区北 13 西 8 ( $\mathbf{T} 060)$

*2 Department of Health Physics, Tokai Research Establishment, Japan Atomic Energy Research Institute; Tokai-mura, Naka-gun, Ibaraki-ken 319-11.

日本原子力研究所東海研究所保健物理部; 茨城県那珂郡 東海村白方白根 2-4 (厂319-11) tissue weighting factors based on the biological information related to the detriment for such as fatal cancer and hereditary disorders.

The effective dose is defined as the sum of weighted equivalent doses in various organs and tissue of the body, and its expression is given below:

$$
E=\sum_{\mathrm{T}} w_{\mathrm{T}} H_{\mathrm{T}}
$$

where $w_{\mathrm{T}}$ is the weighting factor for a tissue $\mathrm{T}$ and $H_{\mathrm{T}}$ is the equivalent dose in the tissue $\mathrm{T}$.

The values of $H_{\mathrm{T}}$ and $E$ are strongly influenced by factors such as the energy of radiation, the orientation of the body in the radiation field and the size of the body. The influence of radiation energy and its geometry on the organ doses and effective dose equivalent, has been revealed from studies by the use of complicated methods such as the Monte Carlo calculations and real phantom 
experiments. $^{2)}$

MARUYAMA, T. et al. have previously studied the relationship between organ doses and filmbadge readings in order to simplify the determinations of the effective dose equivalent. ${ }^{3)}$ In their work, the energy and angular dependences of organ doses were calculated using depth-dose curves experimentally determined with a phantom measurement. Another examples by the same authors $^{4,5}$ ) have been reported where a ratio of the organ dose to the tissue kerma in air for survivors in Hiroshima and Nagasaki was calculated with the mathematical phantoms. While these reports are helpful in estimating the organ doses and in verifying its dependences on the energy and incident angle, the results by using the methodology were available on a limited basis for such specific conditions of external exposure.

SaIto, K. et al. have investigated the effects of body size on organ doses and provided a useful information on their variations that takes into consideration the body weight as the main parameter for organ doses in the cases of environmental $\gamma$ rays. $^{6,7)}$ In order to appropriately determine the organ doses and effective dose, the physical characteristics of the body exposed as well as the source conditions should be considered even with the simplified estimation method. For the simplified estimations of organ doses and effective dose as a whole, it is desired to lead the dominant factor influencing the variations in those quantities and to investigate the effect of the factor in the determination of them.

The authors have introduced an empirical expression for simple determination of organ doses and effective dose equivalent against external photon irradiation. ${ }^{8)}$ In the previous study, only nine male organs were adopted, for simplicity, in calculating effective dose equivalent with the empirical expression applying tissue weighting factors modified for males. In the ICRP Publ. 60, many more organs have been assigned to tissue weighting factors, and calculation method of the remainder dose changed.

At the present, the overall investigations to achieve the simplified estimation method considering the some significant dependences of organ doses and effective dose are rather scarce, particularly for the representative irradiation geometries as described in ICRP Publ. 51 where those geometry conditions are important for the modelling of occupational exposure.

The purpose of the present study aims:

(1) To establish a simplified method which can approximately estimate the effective dose according to new recommendations of the ICRP.

(2) To consider the energy, irradiation geometry and body size dependences of the organ doses and effective dose in an empirical expression.

(3) To confirm the feasibility of approximations in many organs having the different features of its locations in the body.

(4) To represent the specified point in the trunk relevant to the effective dose.

For the application of the method to individual monitoring, the specific point with respect to effective dose will be discussed, in detail, at the effective depth of the body which is determined by taking into account the contribution of the values of tissue weighting factors. The major advantage of effective dose is that the radiation risk for various organs associated with radiation exposure are expressed on a single unified scale.

\section{DETERMINATION OF ORGAN AND EFFECTIVE DEPTHS}

In our simplified expression as a function of depths, it is assumed that the positions of organs in the body, or the distance from the body surface facing an external parallel beam to the organ center principally affect the organ doses and the consequent effective dose. Under the above consideration, the distance between organ center and body surface, namely organ depths $d_{\mathrm{T}}$, were determined by using the organ descriptions given in Cristy' mathematical phantoms ${ }^{9)}$ for the three typical geometries of anteroposterior (AP), posteroanterior (PA) and lateral (LAT) irradiations.

The physical characteristics of the phantoms representing the different body size in ages 1,5 , 10 and reference adults are listed in Table 1. The phantoms consist of three sections representing the main body parts; head, trunk and legs, but the cross-section of the trunk was used to determine the organ depths, since most of the organs necessary to evaluate the effective dose are positioned in the trunk except the thyroid, testes and brain.

The mathematical description of the organs generally uses second order equations: for ex- 
Table 1 Dimensions of phantoms.

\begin{tabular}{lccccc}
\hline A.ge & $\begin{array}{c}\text { Weight } \\
(\mathrm{kg})\end{array}$ & $\begin{array}{c}\text { Height } \\
(\mathrm{cm})\end{array}$ & \multicolumn{2}{c}{ Trunk $(\mathrm{cm})$} \\
\cline { 5 - 5 } & & & $A_{\mathrm{T}}{ }^{* 1)}$ & $B_{\mathrm{T}}{ }^{* 2)}$ \\
\hline Adults & 70 & 174 & 40 & 20 \\
10 years & 30 & 140 & 28 & 16 \\
5 years & 18 & 111 & 22 & 15 \\
1 year & 9 & 78 & 16 & 14 \\
\hline
\end{tabular}

*1) Trunk width from right to left

*2) Trunk depth from front to back

ample, each ovary in adults is an ellipsoid and is defined by this formula:

$$
\left(\frac{x \pm 6}{1}\right)^{2}+\left(\frac{y}{0.5}\right)^{2}+\left(\frac{z-15}{2}\right)^{2} \leq 1
$$

The position of the organs and its shapes were figured in the plane using such equations to determine the organ depths $d_{\mathrm{T}}$ from the trunk surface. The cross-sections of some organs for one year old are depicted in Fig. 1 with the superimposed trunk-contours in the order of increasing age. With such a figure, the dimensions of various organ depth particular to the irradiation geometry and body size were measured in centimeters.

The organs and tissue were selected on the basis of the defined organs and the remainder according to ICRP Publ. 60. In this approximation, the size, shape, composition and density of the organs and tissue are not taken into account but their positions, e.g. the depths from the body surface, are considered.

For the red bone marrow, the depth of a middle spine in the trunk was adopted for the $d_{\mathrm{T}}$, for simplicity, though it is distributed throughout the human body. The bone surface was omitted and

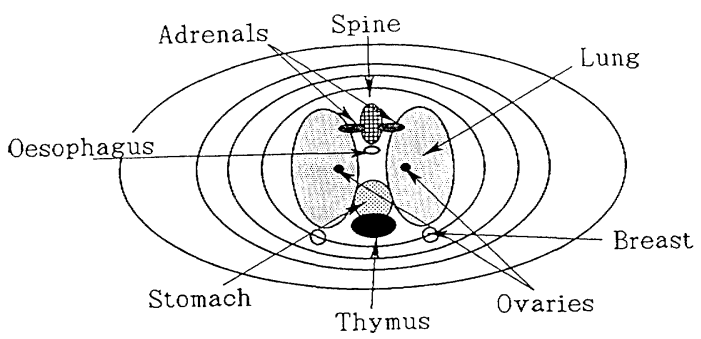

Fig. 1 Superimposed trunk contours of the phantoms used for the adult, 10-year old, 5-year old and 1-year old, with the cross-sections of some organs of 1-year old. its tissue weighting factor $(0.01)$ was added to that of red bone marrow under an assumption that both doses are equal. Similarly, the tissue weighting factor of skin $(0.01)$ was transferred to the gonads, since the expression for approximating the organ doses and effective dose is adequate to the strongly penetrating photon radiation.

An average of two $d_{\mathrm{T}}$ values was taken for paired organs such as the lungs, breasts, ovaries, adrenals, kidneys, testes and thyroids in the determination for the LAT geometry. In that geometry, it is somewhat difficult to determine an appropriate organ depth $d_{\mathrm{T}}$ for some organs located, in particular, close to the front or back surface of the elliptical trunk or outside the trunk.

The ICRP has described an explicit treatment of the remainder for calculating effective dose that in the case when a remainder organ receives an equivalent dose in excess of the twelve defined organs, this organ may be assigned half of the weighting factor for the remainder, with the residual weighting factor applied to the average dose to the rest of the remainder organs. To obtain the remainder's depth, the treatment of $w_{\mathrm{T}} \mathbf{0 . 0 2 5}$ for one of the remainder organs which receives an equivalent dose in excess of the highest dose in any of the defined organs was not applied, because such exceptional cases are considered rare under exposure conditions with an incident beam vertical to the long axis of the body. The upper large intestine and muscle as the remainder were not included in the weighted sum of organ depths due to the difficulty that its center position cannot be expressed as a single point in the body.

Many of the $d_{\mathrm{T}}$ values for 1,5 and 10 years of age for different geometries were determined using mathematical descriptions with the same method for adults. The values of $d_{\mathrm{T}}$ obtained in this way are summarized in Table 2.

If the equivalent doses in many organs for the estimation of effective dose are, as a whole, determined principally by the parameter of depths, it would be possible to define the specific depth relevant to the effective dose with the tissue weighting factors assigned to them. The specific depth with respect to effective dose is named as the effective depth, and is obtained from a sum of weighted $d_{\mathrm{T}}$ values by the same expression as Eq. (1)

$$
d_{\mathrm{E}}=\sum_{\mathrm{T}} w_{\mathrm{T}} d_{\mathrm{T}}(\mathrm{cm})
$$




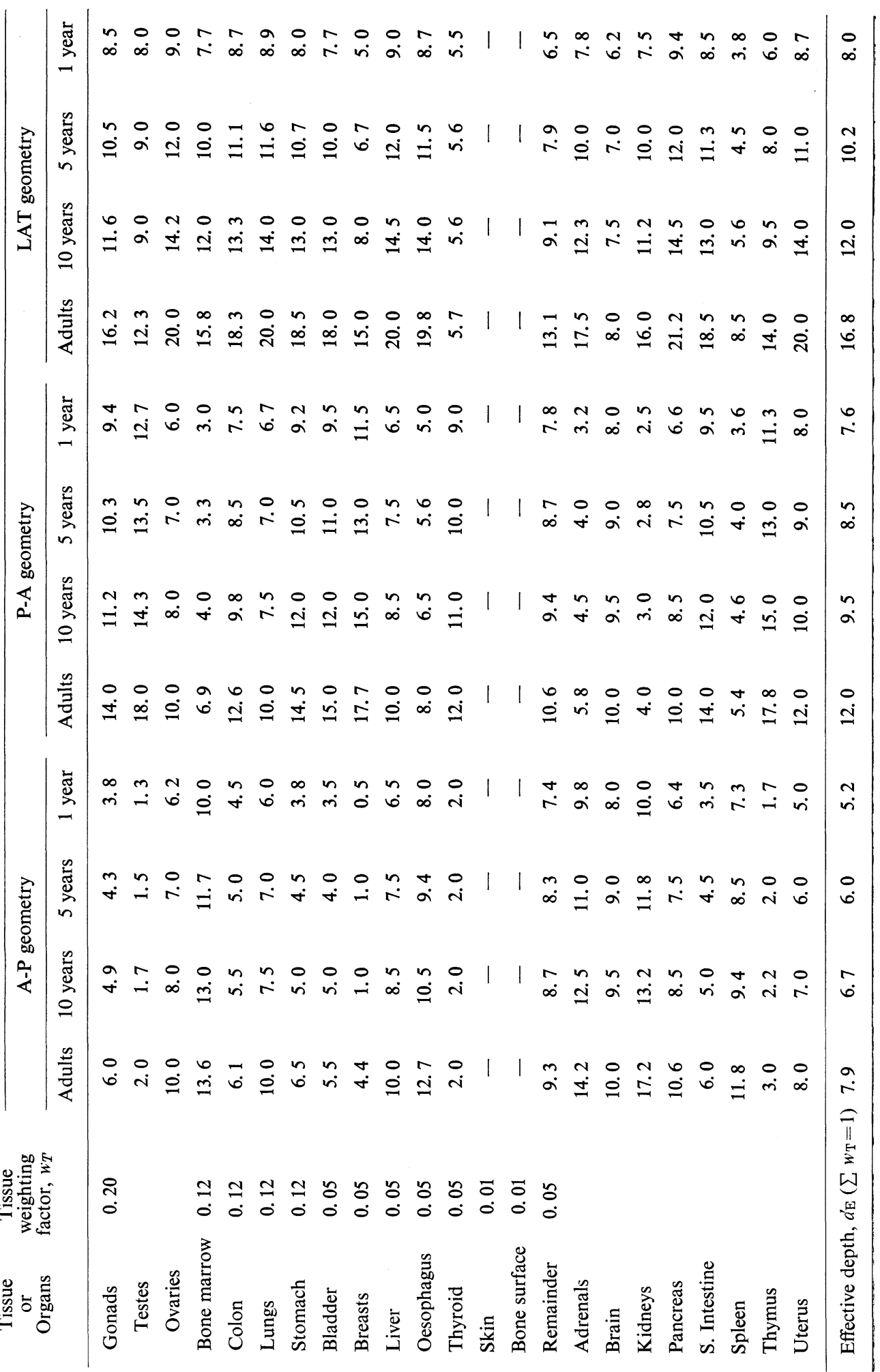


The relationship between organ depth $\left(d_{\mathrm{T}}\right)$ and effective depth $\left(d_{\mathrm{E}}\right)$ mediated through tissue weighting factors $\left(w_{\mathrm{T}}\right)$ was derived from the consideration of the definition of effective dose, i.e. the basic function of $\omega_{\mathrm{T}}$ is to link the various organ doses to the single objective quantity of effective dose.

\section{EQUIVALENT DOSES AS A FUNCTION OF DEPTHS}

The values of organ doses involving effective dose are generally influenced by the geometry of radiation field, its energy and the size or configuration of the body exposed. Those factors' effects on the equivalent doses can be anticipated in accordance with a certain regularity. Approximating the variation of organ doses and effective dose with the depth variable would be useful to understand the feature of effective dose as the objective quantity in the monitoring for the purpose of radiation protection.

The method is based on the assumption that strongly-penetrating photons incident to the body travel in straight lines to the organ center and attenuate exponentially with penetration distance. Hence, the attenuated extent of organ doses can be approximated with an exponential function of the depths for deeps lying organs. The empirical expression for the approximation of the equivalent doses of various organs in the forms of conversion coefficients is

$$
H_{\mathrm{T}} / D_{\mathrm{air}}=A+B \exp \left(-\mu d_{\mathrm{T}}\right) \quad(\mathrm{Sv} / \mathrm{Gy})
$$

In the same way for approximation of the effective dose

$$
E / D_{\mathrm{air}}=A+B \exp \left(-\mu d_{\mathrm{E}}\right) \quad(\mathrm{Sv} / \mathrm{Gy})
$$

where $A$ and $B$ are the coefficients relevant to photon energy 0.015 to $10 \mathrm{MeV}$. While $B$ provide the build-up of equivalent doses, a combination of $A$ with $B$ which function gives appropriate approximations for deeper organs. $\mu$ is the photon mass absorption coefficient for muscles, ${ }^{10)}$ the density of which is approximated as 1 $\mathrm{g} / \mathrm{cm}^{3}$. The coefficients $A, B$ and $\mu$ are listed in Table 3.

To establish this functional form and to obtain $A$ and $B$, an exponential function that approximate organ doses was fitted to the available data of organ doses in ICRP Publ. 51 using the least squares method.

By use of the expression, the authors have previously illustrated the estimates for male
Table 3 The fitted coefficients $A$ and $B$ in Eq. (3).

\begin{tabular}{clrc}
\hline $\begin{array}{c}\text { Photon energy } \\
(\mathrm{MeV})\end{array}$ & $\begin{array}{c}\mu \\
\left(\mathrm{cm}^{2} / \mathrm{g}\right)\end{array}$ & \multicolumn{1}{c}{$\begin{array}{c}A \\
(\mathrm{~Sv} / \mathrm{Gy})\end{array}$} & $\begin{array}{c}B \\
(\mathrm{~Sv} / \mathrm{Gy})\end{array}$ \\
\hline 0.015 & 1.36 & 0.01 & 0.12 \\
0.025 & 0.267 & 0.11 & 0.42 \\
0.050 & 0.0409 & -0.95 & 2.94 \\
0.060 & 0.0312 & -1.37 & 3.48 \\
0.070 & 0.027 & -1.68 & 3.83 \\
0.080 & 0.0255 & -1.67 & 3.78 \\
0.100 & 0.0252 & -1.50 & 3.51 \\
0.150 & 0.0276 & -0.87 & 2.64 \\
0.200 & 0.0297 & -0.50 & 2.11 \\
0.300 & 0.0317 & -0.24 & 1.73 \\
0.500 & 0.0327 & 0.01 & 1.34 \\
1.000 & 0.0308 & 0.15 & 1.10 \\
3.000 & 0.0225 & 0.16 & 1.04 \\
6.000 & 0.0178 & 0.02 & 1.19 \\
10.00 & 0.0154 & -0.09 & 1.32 \\
\hline
\end{tabular}

organs and the effective dose equivalent with the weighting factors modified from those of the ICRP, for the simplification of computation. In this work, the inherent organs in females and other organs were added for complete calculation of the effective dose, based on the new tissue weighting factors described in ICRP Publ. 60.

\section{RESULTS AND DISCUSSION}

\section{Comparison with the precise data}

Many organs and tissues of adults for calculating the effective dose are distributed at various locations with the depth range of 2 to about 20 $\mathrm{cm}$ in the trunk. The results were compared with the precise data to ascertain the feasibility of the simple expression not only for the effective dose but for various organ doses. The approximates for adults and the precise values by the Monte Carlo method agree, in general, within $20 \%$ for the main organs lying deep.

The variation of organ doses due to its locations for external photon exposure can be easily found by the detail comparisons for the femalespecific organs which have relatively different characteristics of positions and shapes from males. Figure 2 (a) (c) show the approximate results compared to the Monte Carlo calculations for some organs in which somewhat different dose values appear for both sexes. 
(a) Gonads
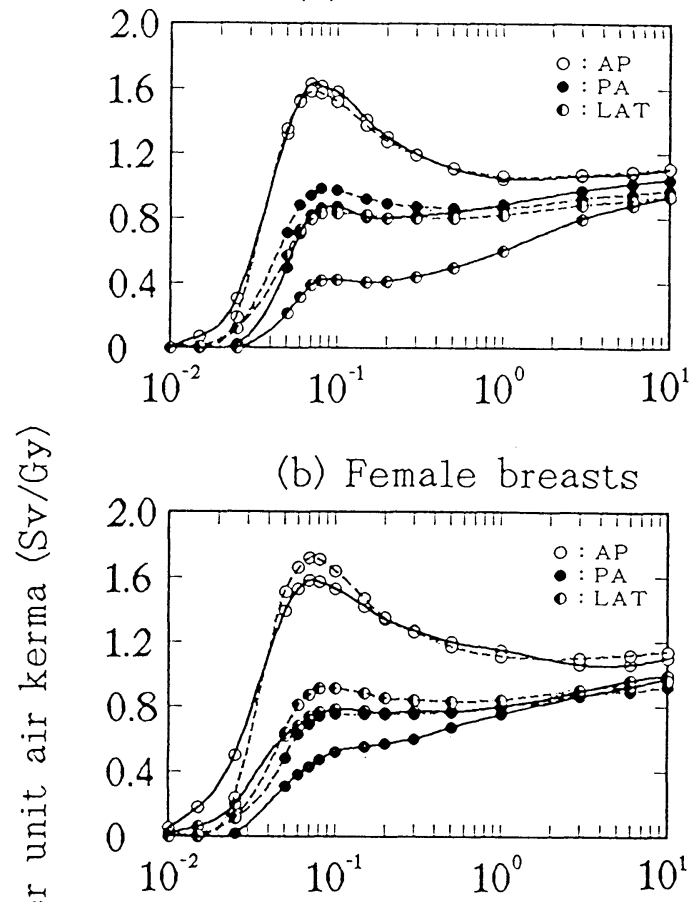

(c) Female lungs

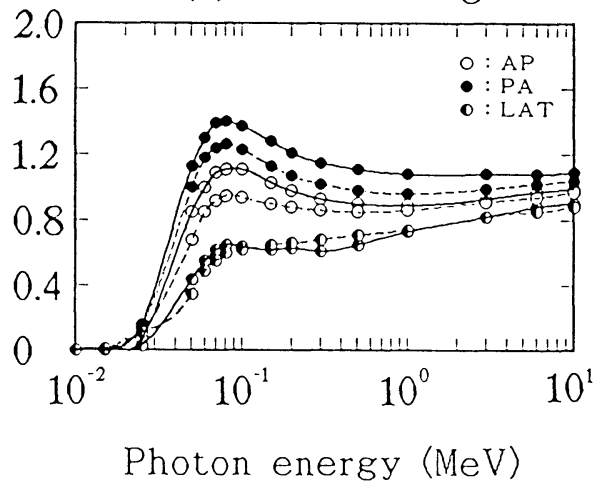

Fig. 2 Approximate doses (broken line) for female organs having different characteristics in both sexes, compared with the calculations (solid line) by the Monte Carlo method for photons incident in AP, PA and LAT geometries.

To obtain the approximate for gonads, an average depth of testes and ovaries was applied to the expression for each geometry. The solid line data shown in the Fig. 2 (a) is also referred to the arithmetric mean of the organ dose values in both sexes given in ICRP Publ. 51. For AP and PA geometry, the simplest averaging of depths over both sexes gives a good approximate for the genitals which vary in positions; the ovaries being situated in the center line and the testes near the front surface of the body.

In the cases of LAT geometry, however, the gonads result calculated with the mean depth of $16.2 \mathrm{~cm}$ shows $50 \%$ overestimate in the energy range of $0.05-1 \mathrm{MeV}$. But, if the average depth of $20 \mathrm{~cm}$ is applied, as the testes depth measured from the point of center-line edge of the elliptical cross-section, the discrepancy with precise data is reduced for about $25 \%$ in that geometry. With the expression overestimate, in general, for some organs located in close proximity to the body surface (testes and thyroids anteriorly and kidneys and adrenals posteriorly) in the case of LAT geometry, since the depths of those organs become shorter with the beam parallel to the center line of the trunk which has a concave shape.

When irradiating from the back, the doses of breasts have lower values than those from front or lateral due to larger shielding effect of the trunk. Similar tendency can be found in female lungs for AP geometry, where breasts shield the lungs, as illustrated in Fig. 2 (b) $\sim(c)$. In the figure, the approximate estimates of female lungs agree well with the precise data for LAT geometry, but the approximates for AP and PA geometries showed an underestimate of about 15 $\%$. The reason may be due to the difference of phantom size used which affects the deviation of both calculations; the approximates obtained from the depths of unisex phantom having reference man size, while the precise data calculated for female phantom have smaller size than the former. That is, the larger a body size is, the lower deep-organ doses are due to the shielding of the body itself. From these features, it can be concluded that variations in organ doses are determined mainly by the organ positions (the depths) for external exposure.

The approximate results of effective doses for adults, were compared with the precise calculations $^{11)}$ by the Monte Carlo method and the MIRD-type phantom for the same set of irradiation geometries and energy range. Figure 3 shows the comparison of effective dose with the two methods.

The approximate values agreed well within 15 $\%$ on average with the precise ones in the energy region for the irradiation geometries of interest in spite of some approximations in determining $d_{\mathrm{T}}$ 


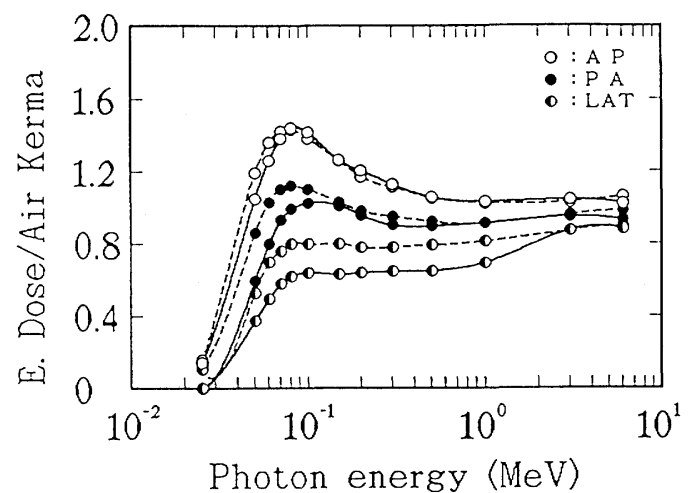

Fig. 3 Comparison of the approximate effective dose $E$ (broken line) per unit air kerma in free air and the precised effective dose calculated by the Monte Carlo method (solid line).

and $d_{\mathrm{E}}$. For the LAT geometry, however, differences between the two results are notably (about $25 \%$ ) for the lateral geometry. By comparing both results, the expression is considered to provide a generally good approximation for organ doses and effective dose.

\section{Dose variation with body size}

With the simple expression, various organ doses and effective dose are easily approximated as a function of the organ depths which vary with the body size. The effect of body size on organ doses for environmental $\gamma$ rays has been investigated by SAITo et al.6); they expressed the variations of organ doses as a function of the body weight

$$
D=a \exp \left(b w^{1 / 3}\right)
$$

where $w$ is the body weight, and $a$ and $b$ are coefficients determined by irradiation conditions and organs. The variable $w^{1 / 3}$, cube root of body weight, is analogous in principal to the organ depth by a dimension of length, since most organs are assumed to consist of soft tissue with the density of $1 \mathrm{~g} / \mathrm{cm}^{3}$.

Both our expression and SAITo's one have adopted the same assumption that organ doses would be approximated by an exponential function of the path length of penetrating $\gamma$ rays, but $a$ and $b$ coefficients work differently in each expression. Our coefficients in Eqs. (4) (5) are determined only by photon energies, but SAITo's coefficients in Eq. (6) are determined by individual organs and irradiation conditions. The results by both methods could not be compared directly because irradiation conditions are significantly different from each other.

Using Eq. (5), different effective doses in age were investigated, which is illustrated in Fig. 4. The effective depths under 10 year-olds were obtained with the tissue weighting factors for adults because these factors are not available for children. In the figure, effective doses for ages of 1,5 , and 10 years were normalized to the values for adults.

It can be seen that the variations of effective

(a) AP Geometry

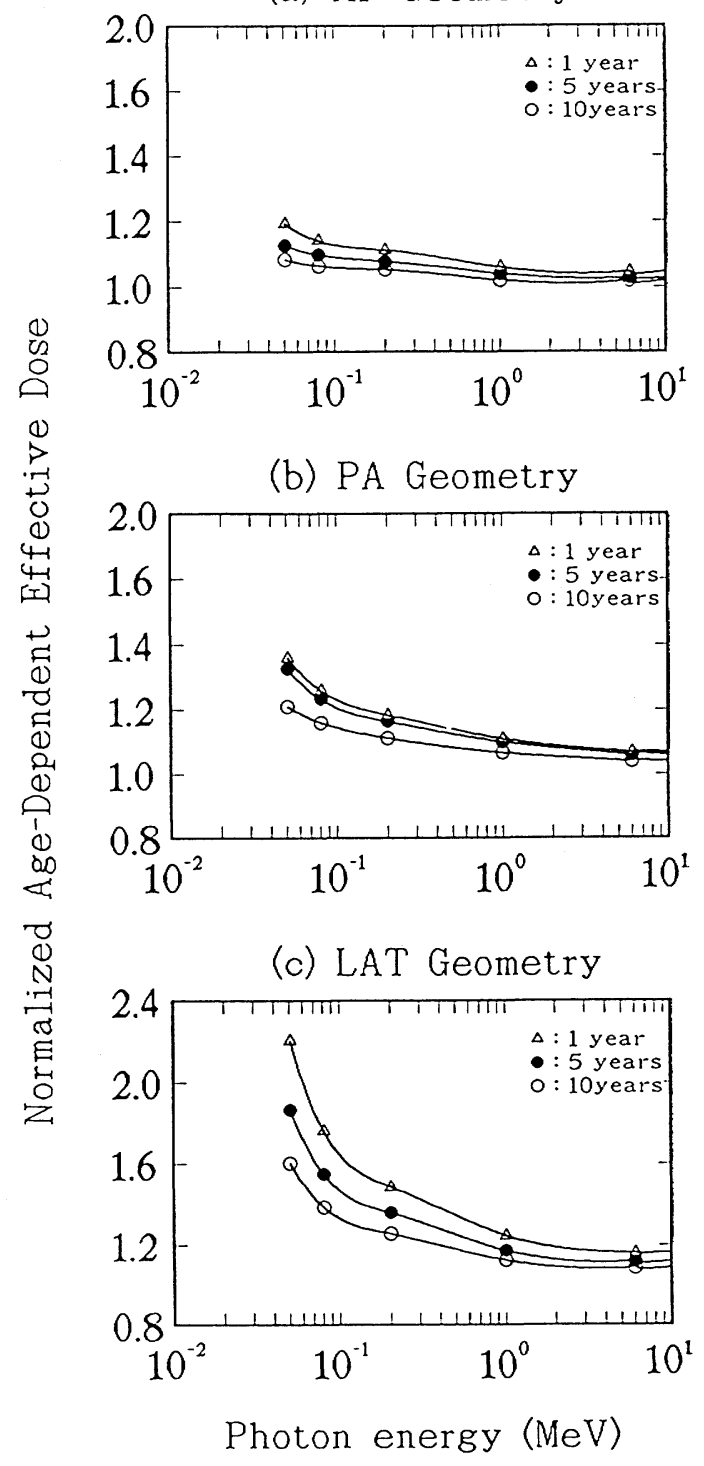

Fig. 4 Age-dependent effective doses normalized to adult doses for various geometries. 
dose in age are strongly dependent on the irradiation geometry due to the difference of organ depth by age. For instance in LAT geometry, the effective dose of 1 year-olds shows a higher value than that of adults by a factor of 2.2 , while for AP geometry by a factor of 1.2. One of the reason for this dependence of irradiation geometry is that the cross-section of children's trunk is more circular than that of adults, as shown in Fig. 1. From this result, the different effective dose by age are considered to be affected by the trunk size and its configuration.

Although it would be difficult to completely explain those variations in age-dependent effective dose only with the variation in body size, however, the concept of organ depth may give a useful information on the changes of organ and effective doses with respect to the body size. It is known that an individual size of the human body is widely distributed even in the same age group. ${ }^{12)}$ Therefore, it would be possible to perceive the variations of effective dose, which reflect the individual characteristics with variation in size, compared to that of the reference man.

The relationship between age-dependent effective depth and the weight of the phantom used is shown in Fig. 5, the poles arrayed along the front of the histogram express the cubic root of the weight at each age, normalized with the adult values. Those arrayed at the back are the agedependent effective depths $d_{\mathrm{E}}$ normalized to the $d_{\mathrm{E}}$ of adults for the ROT, LAT, PA and AP geometries, respectively. The figure shows that the $d_{\mathrm{E}}$ values depending on age are directly propor-

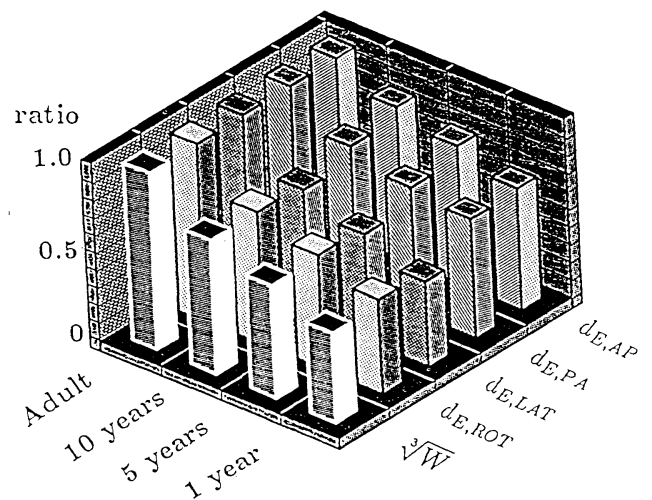

Fig. 5 Histogram of the ratios of age-dependent effective depths for various geometries and the cubic root of the weight of phantoms by age, normalized to the adult values. tional to the cubic root of body weights. Another approach is the use of organ and effective depth which is similar in concept to the approximation of organ doses as a function of body weight.

3. Feasibility of the concept of effective depth

In our simple method, organ and effective doses could be expressed only with the variable of organ depths, which are also simply obtained from organ positions depicted in the cross-sections of the torso. The exact center position of an organ can not be precisely found since its location is not fixed in living human body. Therefore, it would be useless to discuss the precise organ depth, because the coordinate of organ position is assumed to be fixed, and its mathematical description is also an approximation for real organ. However, from the organ depth, an important parameter related to effective dose can be obtained with tissue weighting factors assigned to the organ depths. If the effective depth gives a good approximate for effective dose within allowable errors, it may be possible to define the specific point of effective dose which can be plotted with the effective depth in the trunk.

In practical radiation monitoring, an operational quantity ${ }^{13)}$ of the ambient dose equivalent $H^{*}(10)$ which is defined at a depth of $1 \mathrm{~cm}$ in the ICRU sphere has been used. The use of $H^{*}(10)$ could not be changed with the revision of effective dose which superseded the previous effective dose equivalent. ${ }^{14)}$ Figure 6 is prepared to understand the comparison of the specified point for $H^{*}(10)$ with the point for effective dose determined by the effective depth in this work. As illustrated in the Figure, the specified point for effective dose is located on the point close to the center of elliptical shape phantom (about $2 \mathrm{~cm}$ forward from the center).

KusAmA et al. have proposed, with Mix-DP phantom measurements, that the mid-line dose equivalent express well the effective dose equivalent rather than $1 \mathrm{~cm}$ dose equivalent. ${ }^{15}$ ) The proposal is somewhat apparent considering the locations of organs participating in a weighted sum with its doses.

Since the specified point for effective dose was obtained from the combinations of effective depths for three geometries, the point is seen as a transverse line with long narrow shape. Howerer, the point can be specified as a point taking 


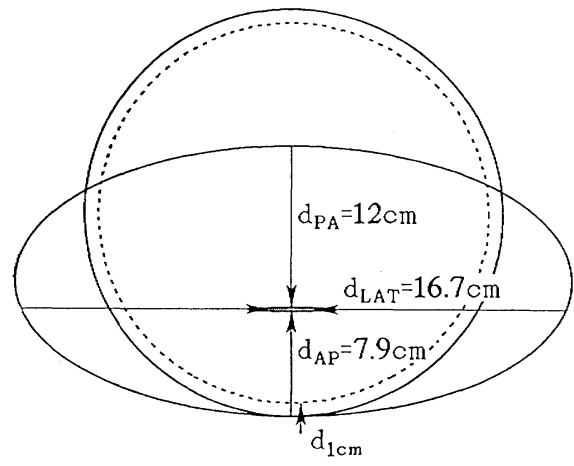

Fig. 6 Description of the specified point of effective depth representing the effective dose in the adult trunk, and the broken circle described for the reference point of $H^{*}(10)$ with a depth $d=1 \mathrm{~cm}$ within a cross-section of ICRU tissueequivalent sphere.

into consideration the uncertainty of the organ depth when determining it for LAT geometry. Considering the concept or meaning of effective depth, in a sense, the point represents the position of a hypothetical organ that includes the whole contributions of tissue weighting factors assigned to many organs in calculating the effective dose.

Therefore, for external photon exposure, it would be possible to calibrate a dosemeter in terms of the equivalent dose to the specified point in an appropriate phantom, so the measuring quantity with the dosemeter can be related to the effective dose as an objective dose in practical monitoring. Further study on this aspect is planned and a report is expected on the results in the future. While the point with respect to effective dose is simply achieved using the parameter of effective depth in this study, other new approaches are desired to find the specified point for effective dose, and to ascertain the feasibility of effective depth-with experimental or computational methods.

\section{CONCLUSION}

The purpose of this study was to establish a simplified method for approximating organ doses and effective dose, and to verify the features of effective dose with the effective depth. Many organs and effective doses agree well with the precise data calculated by Monte Carlo techniques within about $20 \%$, with the exception of some organs located adjacent to the body surface in LAT geometry. It could be concluded that the simple expression provides a generally good approximation for effective dose, and that variations in organ doses are determined mainly by the organ positions, consequently the organ depths, for external photon exposure.

Age-dependent effective doses were investigated with different effective depths by age; the doses also varied with irradiation geometry. It was found that the variations of effective dose in age and smaller for the AP geometry and larger for the LAT geometry, since most of the organs considered in effective dose are located in front of the body. The results for the ages below adults were not compared with the precise data by the Monte Carlo method due to unavailability of data. It is thus hard to explain that variations in age-dependent effective dose are determined mainly by the change of body size. However, the concept of organ depth may be normally adequate, compared to another method in which various organ doses can be approximated as a function of the body weight.

Using the effective depth, the specified point for effective dose could be determined at a depth of about $8 \mathrm{~cm}$ from the front surface in the body with the shape of elliptical cylinder. The point is considered to represent the position of a hypothetical organ that includes the contributions of tissue weighting factors assigned to many organs, and it would be possible to calibrate a dosemeter in terms of the point dose for effective dose in an appropriate phantom.

\section{REFERENCES}

1) ICRP Publication 60 (1991).

2) ICRP Publication 51 (1987).

3) T. Maruyama, K. Nishizawa, Y. Noda, E. TAKeda, K. Fukuhisa, Y. Furuya and T. HaSHIZUME; Estimation of population doses and risk estimates from occupational exposure in Japan, 1978 Part 1. Determinations of organ or tissue doses and effective dose equivalents, $J$. Radiat. Res., 22, 182 (1981).

4) T. Hashizume, T. Maruyama, K. Nishizawa, Y. Noda, K. Fukuhisa and E. Takeda; Determinations of organ or tissue doses to survivors in Hiroshima and Nagasaki, J. Radiat Res., 21, 213 (1980).

5) T. Hashizume, T. Maruyama, K. Nishizawa and A. Nishimura; Dose estimation of human fetus exposed in utero to radiations from atomic bombs in Hiroshima and Nagasaki, J. Radiat. Res., 14, 346 (1973). 
6) K. Saito, N. Petoussi, M. Zankl, R. Veit, P. JACOB and G. DreXler; Organ doses as a function of body weight for environmental gamma rays, J. Nucl. Scien. Tech., 28, 627 (1991).

7) N. Petoussi, P. JACOB, M. ZANKL and K. Saito; Organ doses for foetuses, babies, children and adults from environmental gamma rays, Radiat. Prot. Dosim., 37(1), 31 (1991).

8) S. SA and M. NARITA; Simple empirical expression to evaluate effective dose equivalent for external photon irradiation, J. Nucl. Scien. Tech., 29, 396 (1992).

9) M. CRISTY; Mathematical phantoms representing children of various ages for use in estimates of internal dose, NUREG/CR-1159 (1980).
10) ICRU Report 10b, NBS Handbook 85 (1962).

11) Y. YAMAGUCHI; Calculation of effective dose for external photon exposure based on ICRP's new recommendation (in Japanese), RADIOISOTOPES, 40, 459 (1991).

12) G. TANAKA; Japanese reference man (in Japanese), Hoken Butsuri, 25, 49 (1990).

13) ICRU Report 39 (1985).

14) ICRP Publication 26 (1977).

15) T. Kusama, K. Sugai and Y. Yoshizawa; Experimental approach to application of effective dose equivalents, one-cm-depth dose equivalent and mid-line dose equivalent to radiation protection practice (in Japanese), Hoken Butsuri, 18, 353 (1983). 